\title{
Metabolic changes in Asian Muslim pregnant mothers observing the Ramadan fast in Britain
}

\author{
By ASHOK MALHOTRA ${ }^{1}$, P. H. SCOTT ${ }^{2}$, J. SCOTT ${ }^{2}$, H. GEE ${ }^{1}$ \\ AND B. A. WHARTON ${ }^{1 * \dagger}$ \\ ${ }^{1}$ Sorrento Maternity Hospital, Birmingham B13 $9 H E$ and ${ }^{2}$ Biochemistry Department, \\ Selly Oak Hospital, Birmingham B29 6JD
}

(Received I June 1988-Accepted 5 January 1989)

\begin{abstract}
1. Metabolic changes associated with Ramadan fasting were studied in eleven Asian pregnant mothers. This was compared with a group of control mothers undergoing a normal physiological fast.

2. At the end of the Ramadan fast day there was a significant fall in glucose, insulin, lactate and carnitine, and a rise in triglyceride, non-esterified fatty acid and 3-hydroxybutyrate. When compared with the control group, none of the Ramadan mothers had a completely normal set of biochemical values at the end of the fast day.

3. Pregnancy outcome in the two groups was comparable.

4. We are wary of the metabolic departures from normal observed in the Ramadan fasting mothers. If asked we advise mothers to take up the dispensation from fasting during pregnancy which is allowed.
\end{abstract}

An important aspect of the Muslim religion is the fast of Rosa during Ramadan. During Ramadan all healthy adults are obliged to fast from sunrise to sunset. The length of the fast therefore depends on the exact time of sunrise and sunset in the country where the person lives. The duration of the fast in temperate climates such as Britain averages about $18 \mathrm{~h} / \mathrm{d}$ when Ramadan occurs during the spring and summer. This is dependent on the exact time of sunrise and sunset, but is approximately observed between 02.30 and 19.30 hours here. Pregnant women are allowed to postpone their fast until after delivery, but about three quarters of mothers attending this hospital continue to observe it for various reasons (Eaton \& Wharton, 1982), for example, convenience when eating as a family, and social pressures. However, they often ask about the possible harmful effects of fasting both to themselves and their babies.

Although there is some evidence of metabolic stress in Ramadan (Prentice et al. 1983), the work was carried out in the deprived conditions of the tropics where the prevalence of endemic malnutrition, tropical infection and drought may have compounded the effect of fasting during pregnancy. The better environmental conditions of the Muslim population in Britain provides an opportunity to study the metabolic effect of Ramadan fasting without these complicating factors. Moreover, a previous study in Birmingham of nonpregnant Muslim diabetics (Barber et al. 1979) concluded that fasting was safe. This study was designed to determine the metabolic effects of Ramadan fasting during pregnancy in healthy mothers living in Britain.

\section{METHODS}

\section{Patients}

As part of a larger study of glucose metabolism in pregnancy all mothers booking at this hospital in time were invited to attend a clinic at 17 weeks and again at 28 weeks for a more detailed assessment than is usually possible in a routine antenatal clinic. Some of these mothers were selected to take part in this Ramadan study as follows.

* Present address: Department of Human Nutrition, University of Glasgow, Glasgow.

+ For reprints. 
Ramadan fasting $(R F)$ group. As Ramadan approached (29 April-28 May 1987) all Pakistani and Bangladeshi Muslim mothers (forty-four) were asked whether they planned to fast. Eleven failed to reply initially (on subsequent inquiry six fasted and five did not; none were included in the study). Nineteen were not fasting, leaving fourteen fasting mothers who agreed to take part in the study. One of the fourteen Ramadan fasting mothers did not achieve a triceps skinfold thickness increment of $3 \mu \mathrm{m} / \mathrm{d}$, suggesting she had an abnormal pregnancy (Bissenden et al. 1981; Viegas et al. 1987), one mother abandoned the fast and one was shown to have gestational diabetes. These three were therefore excluded from the group. This left eleven in the RF group, i.e. mothers who (1) were Pakistani or Bangladeshi Muslims and observed the Ramadan fast, (2) by 28 weeks were experiencing an apparently normal pregnancy and (3) consented to two venepunctures during $1 \mathrm{~d}$ of Ramadan. The length of the fast was dependent on the exact time of sunrise and sunset. During May 1987 the fasting period was approximately from 02.30 to 19.30 hours.

Overnight fasting $(O N F)$ group. The choice of controls was not straightforward. From the mothers included in the larger study of glucose metabolism (i.e. booked in time for detailed assessments at 17 and 28 weeks) we selected the following mothers who (1) were Pakistani and Bangladeshi Muslims but were not observing the Ramadan fast, (2) at 28 weeks were experiencing an apparently normal pregnancy and (3) consented to metabolic investigation following an overnight fast at 29 weeks. Eleven mothers were recruited.

The biochemical results presented for the ONF group were therefore all from women at 29 weeks of pregnancy whereas the results for the RF group were from the various stages of pregnancy when Ramadan occurred (at 28-37 weeks).

Details of the mothers in the RF and ONF groups are shown in Table 1.

The ONF-group mothers were on average $50 \mathrm{~mm}$ taller than the RF group and of course there was a difference in gestational age when the Ramadan fast occurred. As far as we can see, these differences occurred by chance. They cannot be explained by any unsuspected bias in selection.

\section{Procedures}

Clinical and anthropometric. All mothers attended at 17 and 28 weeks of pregnancy and routine obstetric assessment including a fetal biparietal diameter and maternal anthropometry were carried out. At birth babies were weighed by a midwife and 12-48 h after birth the following additional measurements were made by the first author (A.M.): crown-heel length, mid-biceps and mid-triceps skinfold thicknesses, mid-upper arm circumference of the right arm. Gestational age was calculated from the mothers' last menstrual period unless there was a discrepancy of more than 1 week with the ultrasound readings, in which case the ultrasound dates were taken.

Biochemical. Two fasting samples of blood were obtained from the eleven RF mothers: (a) at the antenatal clinic in the morning (10.50-11.45 hours), following a fast of $8-14 \mathrm{~h}$ timed from the last meal before sunrise; $(b)$ at home in the evening (18.25-20.03 hours), following a fast of 15-22 $\mathrm{h}$ and before the first meal after sunset. One sample was taken from the ONF mothers at $09.00-10.00$ hours at the antenatal clinic following a fast of 9-14 h.

Blood specimens collected at the hospital were processed immediately, those collected at home were transported in an ice-cooled vacuum flask and, apart from precipitation of the 3-hydroxybutyrate-lactate-pyruvate sample which was done immediately, were processed within 10 min. Venous blood was divided between potassium oxalate-sodium fluoride, lithium heparin, potassium EDTA-trasylol, and plain clotted blood. A sample was taken immediately from the lithium heparin specimen and precipitated with perchloric acid for measurement of 3-hydroxybutyrate, lactate and pyruvate. All specimens were centrifuged 
Table 1. Details of mothers studied

(Mean values and standard deviations for eleven mothers per group)

\begin{tabular}{|c|c|c|c|c|}
\hline & \multicolumn{2}{|c|}{$\begin{array}{l}\text { Ramadan fast } \\
\text { group }\end{array}$} & \multicolumn{2}{|c|}{$\begin{array}{l}\text { Overnight fast } \\
\text { group }\end{array}$} \\
\hline & Mean & SD & Mean & SD \\
\hline \multicolumn{5}{|l|}{ Country of birth } \\
\hline Pakistan & 10 & & 8 & \\
\hline Britain & 0 & & $3^{*}$ & \\
\hline Other & $1 \dagger$ & & 0 & \\
\hline Smokers & 0 & & 0 & \\
\hline \multicolumn{5}{|l|}{ Present pregnancy } \\
\hline Age (years) & $26 \cdot 7$ & $4 \cdot 1$ & $26 \cdot 6$ & $7 \cdot 1$ \\
\hline Height (m) & $1 \cdot 55$ & 0.06 & $1 \cdot 60$ & 0.05 \\
\hline Primipara & 2 & & 4 & \\
\hline Hypertension & 2 & & 1 & \\
\hline Anaemia & 1 & & 1 & \\
\hline \multicolumn{5}{|l|}{ At 28 weeks gestation } \\
\hline Wt $(\mathrm{kg})$ & $63 \cdot 1$ & $9 \cdot 5$ & $66 \cdot 6$ & $9 \cdot 3$ \\
\hline Triceps skinfold (mm) & $21 \cdot 7$ & $6 \cdot 7$ & $20 \cdot 1$ & $6 \cdot 2$ \\
\hline Haemoglobin $(\mathrm{g} / \mathrm{l})$ & 121 & 12 & 122 & 12 \\
\hline \multicolumn{5}{|l|}{ Change between 17 and 28 weeks } \\
\hline Wt velocity $(\mathrm{kg} /$ week $)$ & 0.39 & 0.16 & 0.42 & 0.23 \\
\hline $\begin{array}{l}\text { Triceps skinfold thickness } \\
\text { velocity }(\mu \mathrm{m} / \mathrm{d})\end{array}$ & $33 \cdot 1$ & $12 \cdot 9$ & $24 \cdot 6$ & $10 \cdot 8$ \\
\hline $\begin{array}{l}\text { Gestation at time of metabolic } \\
\text { assessment (weeks) }\end{array}$ & $3 \mathrm{I} \cdot 8$ & 3.7 & $29 \cdot 1$ & $0 \cdot 9$ \\
\hline
\end{tabular}

* Pakistani origin. $†$ Bangladeshi.

and plasma, serum or the supernatant fraction separated. They were stored at either $-20^{\circ}$ or $-70^{\circ}$ as appropriate.

Oxalate-fluoride plasma was used for measurement of glucose using a Model GA1120 Glucose Analyser (Kyoto Company Ltd, Japan). Lithium heparin plasma was used for measurement of triglyceride (using enzymic hydrolysis followed by glycerol kinase, $E C$ 2.7.1.30), carnitine (using carnitine acetyltransferase (EC 2.3.1.7) in an adaptation of the method of Secombe et al. (1978)), and non-esterified fatty acid (using acyl-CoA synthetase (EC 6.2.1.2, Wako Chemical Industries, Japan)), all performed on the Cobas Mira (Roche Diagnostics Ltd). Potassium EDTA-trasylol plasma was used for measurement of insulin (radioimmunoassay/magnetic antibody immunoassay (RIA/MIA), Serono Diagnostics Ltd) and glucagon (RIA assay, Serono Diagnostics Ltd). Blood lactate, pyruvate, and 3-hydroxybutyrate were all measured fluorometrically in supernatant fractions using the method of Maughan (1982).

Results are presented for all measurements on RF patients but not on all ONF patients due to technical reasons.

\section{Ethical considerations}

The investigation was approved by the ethical committee of South Birmingham Health Authority.

$$
\text { Analysis of results }
$$

The women in the RF group acted to some extent as their own controls. Analyses are presented comparing results in the same patients for the first blood sample (RF1, 8-14 $\mathrm{h}$ 


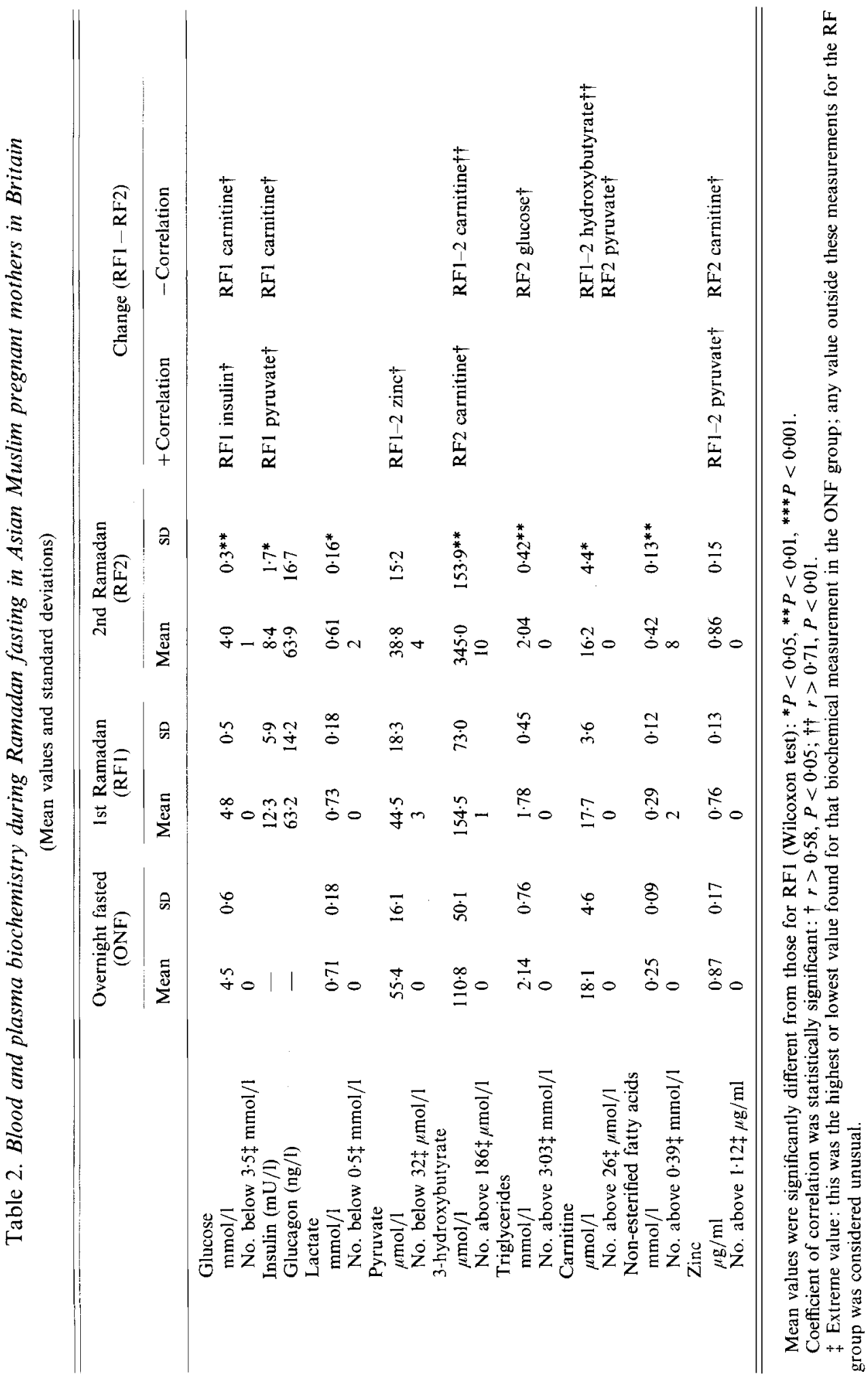



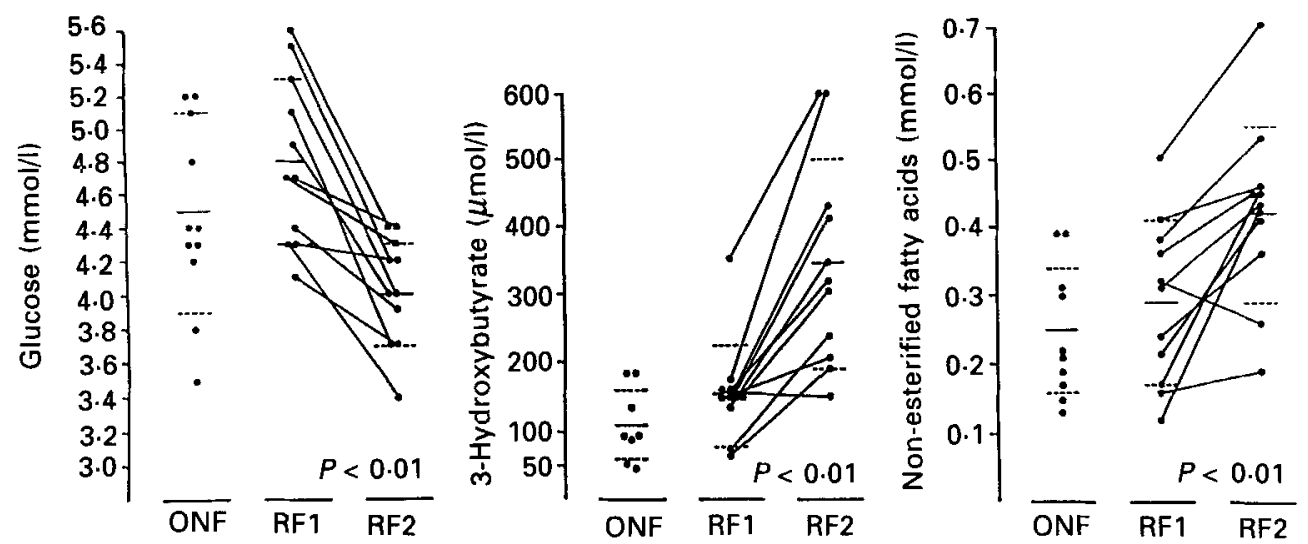

Fig. 1. Changes in blood biochemistry in Asian Muslim women in Britain during the Ramadan fast day (RF group), and in other pregnant patients following an overnight fast (ONF group). ONF group, blood levels after a physiological overnight fast in eleven mothers; RFl group, blood levels after the last meal before sunrise ( $8-14 \mathrm{~h}$ fasting) in eleven mothers; RF 2 group, blood levels before the first meal after sunset (15--22 h fasting) in eleven mothers. Mean values and standard deviations are represented by horizontal bars.

fast) and the second sample (RF2, 15-22 h fast) using mostly paired $t$ and Wilcoxon tests for determination of statistical significance. Analyses are also presented comparing results in the independent RF and ONF groups using independent $t$ and Mann-Whitney tests as indicated in the results section. Regression analyses were performed by the method of least squares.

\section{RESULTS}

Biochemical findings. During the Ramadan fast day plasma glucose, insulin, carnitine and lactate fell (Table 2), while 3-hydroxybutyrate, triglycerides and non-esterified fatty acids rose (Fig. 1). At the end of the Ramadan fast no mother had all plasma results within the physiological fast range, i.e. within the range in the ONF group. One had a lower blood glucose, ten had a higher 3-hydroxybutyrate, two had a lower lactate, four had a lower pyruvate and eight had a higher non-esterified fatty acid.

No biochemical results from patients in the RF group, either at the end of the fast day or in terms of change during the fast day, correlated significantly with gestational age.

Clinical outcome. The groups are too small to make valid comments regarding clinical outcome. There were no significant differences in the size of the babies in the ONF and RF groups although the RF group were on an average $250 \mathrm{~g}$ heavier (Table 3 ). When the mothers who had two or more biochemical abnormalities outside the ONF range were considered, there were no differences in the pregnancy outcome.

Biochemical relations with mother and baby. There were a number of significant correlations between the clinical and biochemical findings in the RF group (Table 4, Fig. 2). For example heavier mothers had higher insulin levels ( $r 0.86)$ but lower carnitine levels $(r-0.68)$ initially, and they experienced greater falls in glucose $(r 0.74)$ and insulin ( $r$ 0.88) during the fasting day; birth weight corrected for sex, gestational age, parity and maternal height (Altman \& Coles, 1980; Thompson et al. 1968) was inversely related to plasma insulin and lactate at the end of the fast day $(r-0.71, P<0.01$, in each case). Similar significant correlations were observed between maternal skinfold thickness and the same biochemical measurements. 
Table 3. Details of pregnancy outcome in Asian Muslim women observing the Ramadan fast in Britain

(Mean values and standard deviations for eleven mothers per group)

\begin{tabular}{|c|c|c|c|c|}
\hline & \multicolumn{2}{|c|}{$\begin{array}{l}\text { Ramadan fast } \\
\text { group }\end{array}$} & \multicolumn{2}{|c|}{$\begin{array}{l}\text { Overnight fast } \\
\text { group }\end{array}$} \\
\hline & Mean & SD & Mean & SD \\
\hline Gestation (weeks) & $39 \cdot 6$ & $1 \cdot 0$ & $39 \cdot 4$ & $1 \cdot 0$ \\
\hline $\begin{array}{l}\text { Birth wt (g) } \\
\text { SD score* } \\
\text { No. below } 10 \text { th centile* }\end{array}$ & $\begin{array}{c}3355 \\
0.07 \\
0\end{array}$ & $\begin{array}{l}256 \\
0.52\end{array}$ & $\begin{array}{c}3100 \\
-0 \cdot 58 \\
2\end{array}$ & $\begin{array}{l}429 \\
0.95\end{array}$ \\
\hline $\begin{array}{l}\text { Apgar score }+ \\
1 \text { min } \\
5 \mathrm{~min}\end{array}$ & $\begin{array}{r}8 \\
10\end{array}$ & $\begin{array}{l}1 \\
0\end{array}$ & $\begin{array}{r}9 \\
10\end{array}$ & $\begin{array}{l}1 \\
1\end{array}$ \\
\hline $\begin{array}{l}\text { Head circumference }(\mathrm{mm}) \\
\text { Length }(\mathrm{mm}) \\
\text { Triceps }(\mathrm{mm}) \\
\text { Biceps }(\mathrm{mm}) \\
\text { Mean upper arm } \\
\text { circumference }(\mathrm{mm})\end{array}$ & $\begin{array}{l}348 \\
502 \\
3 \cdot 6 \\
3 \cdot 1\end{array}$ & $\begin{array}{c}10 \\
19 \\
0.6 \\
0.5\end{array}$ & $\begin{array}{l}345 \\
495 \\
3 \cdot 5 \\
3 \cdot 0\end{array}$ & $\begin{array}{c}9 \\
10 \\
0 \cdot 8 \\
0.6\end{array}$ \\
\hline Congenital abnormalities & $1 \dagger$ & & 0 & \\
\hline Neonatal deaths & 0 & & 0 & \\
\hline Placental wt (g) & 596 & 66 & 537 & 117 \\
\hline
\end{tabular}

* Weight for gestational age and sex of baby, parity and height of mother (Thompson et al. 1968; Altman \& Coles, 1980).

$\dagger$ Adrenogenital syndrome 3- $\beta$-hydroxysteroid dehydrogenase deficiency.

¥ Apgar score: a quantitative rating test with a maximum of ten used to measure the vital signs of a newborn a minute or so after birth: a score greater than seven signifies good health.

\section{DISCUSSION}

Fasting during Ramadan is from sunrise to sunset. The period of starvation may be particularly long for Muslims living in Northern latitudes if Ramadan falls during the summer months. Evidence from Saudi Arabia (Frost \& Pirani, 1987) is that during Ramadan there is a reduction in feeding frequency but that each meal is nutritionally more dense than that taken outside Ramadan. A previous study from this hospital showed that some mothers actually increased their intake during Ramadan (Eaton \& Wharton, 1982). Even so, we did not find any significant differences in biochemical results between the RF1 and the ONF results following a fast of up to $14 \mathrm{~h}$.

In the present study starvation due to the Ramadan fast lasting up to $22 \mathrm{~h}$ occurred, which is much longer than a normal overnight fast, and is also, importantly, much longer than the Ramadan fast experienced in the geographical proximity of the equator. This prolonged fasting may have biochemical and clinical implications.

Biochemical implications. During the initial period of the fasting day the biochemical findings were the same as those seen following an overnight fast, but thereafter as the fasting day continued there were falls in glucose, insulin, lactate and carnitine and rises in 3-hydroxybutyrate, triglyceride and non-esterified fatty acids. Excluding the fall in carnitine, these changes commonly accompany prolonged fasting in non-pregnant individuals, and some of them have also been described in pregnant women - in Gambian women during Ramadan (Prentice et al. 1983) and in mothers fasted up to $84 \mathrm{~h}$ before an 


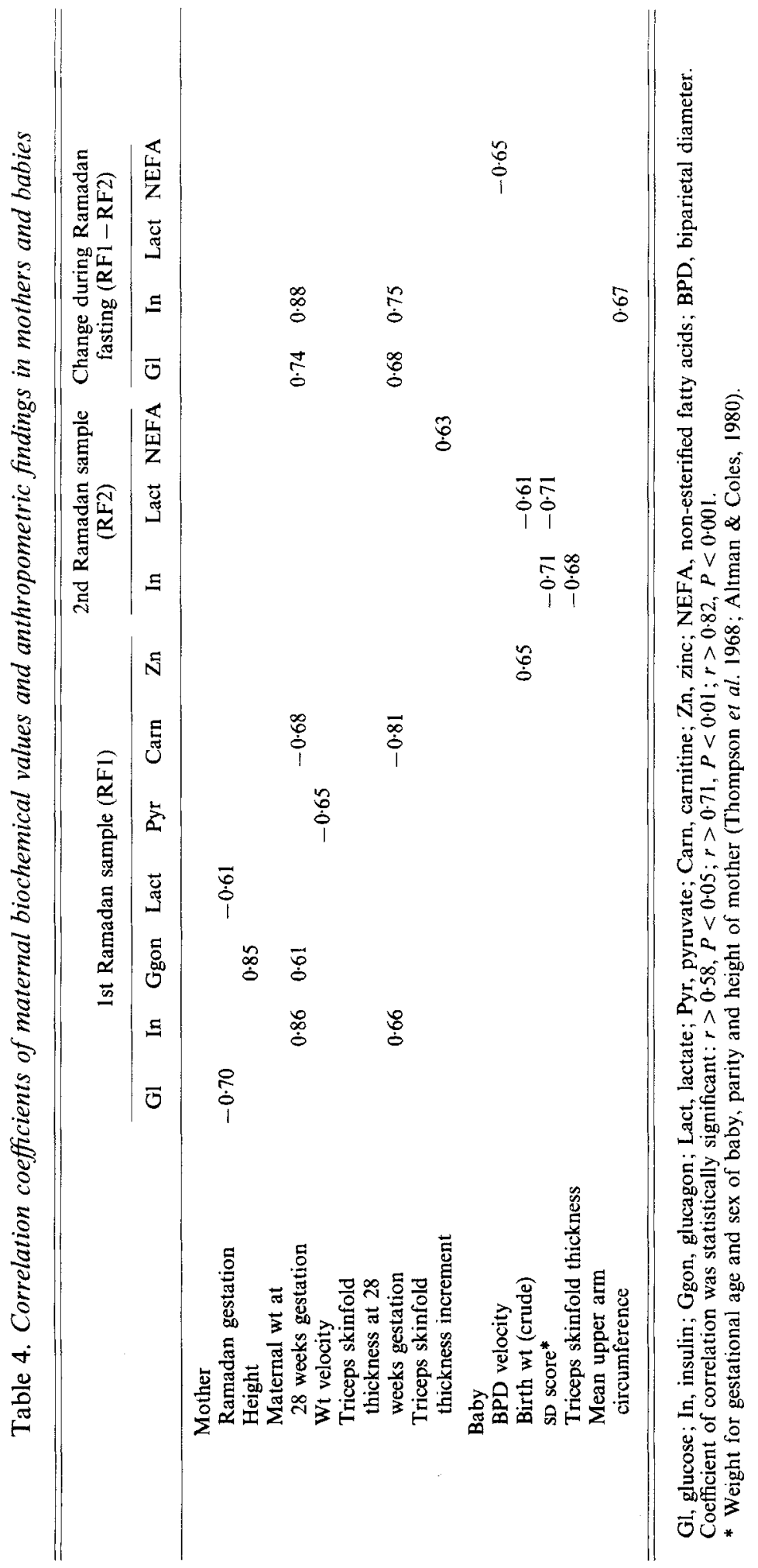



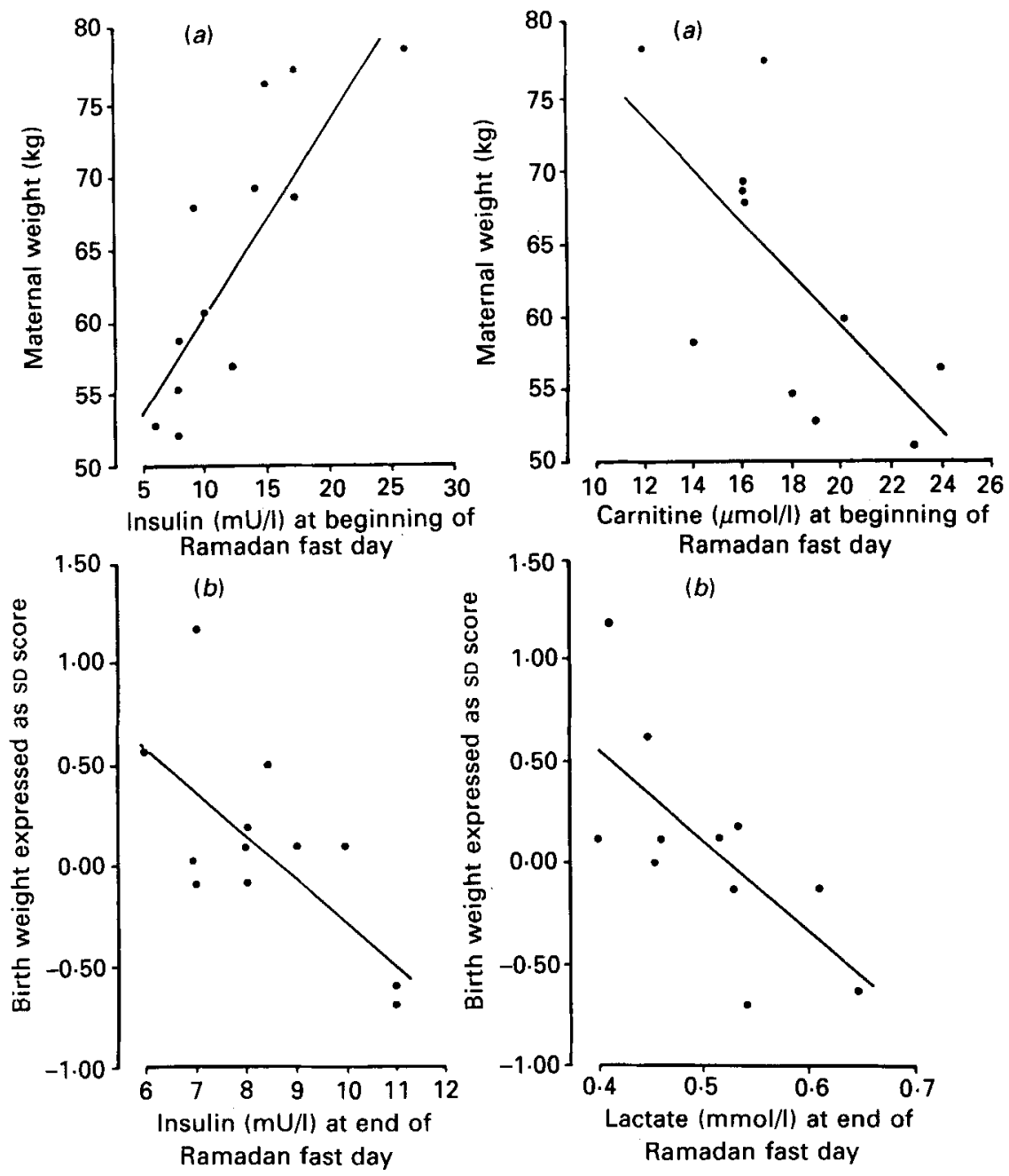

Fig. 2. Correlations between maternal biochemical values in Asian Muslim women in Britain observing the Ramadan fast and anthropometric findings in mothers and babies. (a) Maternal weight at 28 weeks gestation and insulin $(r 0.86, P<0.01)$ and carnitine $(r-0.68, P<0.05)$ at the beginning of the Ramadan fast day. (b) Birth weight expressed as standard deviation (SD) score and insulin and lactate $(r-0.71, P<0.01)$ at the end of the Ramadan fast day.

elective termination (Felig \& Lynch, 1970; Tyson et al. 1976). The changes in carnitine have not been described previously. A reduction in plasma carnitine concentration during pregnancy has been observed elsewhere (Bargen-Lockner et al. 1981) and has been shown to involve both free and acyl carnitine. It has been suggested that this may result from active transfer of carnitine from the mother to the fetus but this requires confirmation. We measured only free carnitine but showed that, although it was already low, it fell further during the Ramadan fasting day.

Carnitine is widely distributed throughout the tissues of the body and is necessary for the transport of long-chain non-esterified fatty acids across the mitochondrial membrane. Only after entry into the mitochondrion can fatty acid oxidation and ketogenesis occur. If, due to lack of carnitine, this is prevented, then lipid becomes deposited in many tissues, a 
common finding in inherited systemic carnitine deficiency (Angelini et al. 1987). Individuals suffering from this disease show on prolonged fasting an expected fall in blood sugar and steady increases in both plasma triglyceride and non-esterified fatty acid, but fail to initiate a significant ketogenesis (Chapoy et al. 1980). Levels of serum carnitine measured in these patients are very much lower than those found in pregnancy. However in our prolonged Ramadan fasted mothers we found evidence that they may be demonstrating similar although much milder changes. Accompanying a fall in plasma glucose there was a steady increase in both serum triglyceride and non-esterified fatty acid, yet though 3hydroxybutyrate also increased, there was a significant correlation indicating that mothers with low plasma carnitine showed a much smaller increase in ketone production than did mothers with high plasma carnitine. At the same time, we also found negative correlations between plasma carnitine and both maternal weight and triceps skinfold thickness at 28 weeks gestation. These observations suggest that the apparent carnitine deficiency seen during pregnancy may be an important factor in minimizing metabolic use of fat whilst encouraging maternal fatness.

The fall in plasma glucose seen during the Ramadan fast was also associated with a fall in serum insulin. Insulin is antilipolytic and therefore this decrease would be expected to be accompanied by the increases in serum triglyceride and non-esterified fatty acid already noted. We would have expected to see a concomitant rise in serum glucagon but this did not occur. Absence of such a rise may have blunted the ketogenic effect of a decrease in the insulin: glucagon ratio, as we found no relations here with ketone production. Insulin was, however, positively correlated with maternal weight and triceps skinfold thickness at 28 weeks' gestation, indicating the expected influence of insulin on maternal fatness.

So both low carnitine and high insulin work together to promote fatness in these Asian mothers, whilst limiting use of fat for energy metabolism. The negative correlation between the two measurements in the first Ramadan sample just failed to reach statistical significance. However, it is interesting that there were strong negative correlations suggesting that low serum carnitine accompanied a greater fall in both glucose and insulin during the Ramadan fast. Thus, in the presence of low carnitine, fat which was mobilized as the fast progressed was not utilized by the mother, but was perhaps directed to the growing fetus.

Clinical implications. Prentice et al. (1983) described a poor obstetric outcome in the Ramadan fasting group in The Gambia but the outcome in their control group was not stated. In our study the outcome of pregnancy in this small group of mothers was satisfactory and no different from the control group. Nevertheless, the biochemical changes were outside the range seen following a normal fast: no mother had a completely normal set of biochemical values at the end of the Ramadan fast day. We also note that the higher values for insulin and lactate seen at the end of the Ramadan fast day were associated with lighter, thinner babies. Although we did not observe any clinical harm, we are nevertheless wary of these metabolic departures from normal and we consider it prudent to recommend that mothers take up the dispensation offered to them during Ramadan, i.e. postpone their observation of the fast until after pregnancy is completed.

A. M. was the Mary Crosse research fellow. Sister T. Douse and Mrs P. Wharton helped in the metabolic clinics. Serono Ltd provided the radioimmunoassay kits. 


\section{REFERENCES}

Altman, D. G. \& Coles, E. C. (1980). Nomograms for precise determination of birth weight for dates. British Journal of Obstetrics and Gynaecology 87, 81-86.

Angelini, C., Trevisan, C., Isaya, G., Pegolo, G. \& Vergani, L. (1987). Clinical varieties of carnitine and carnitine palmitoyltransferase deficiency. Clinical Biochemistry 20,1-7

Barber, S. G., Fairweather, S., Wright, A. D., Fitzgerald, H. G. \& Malins, J. M. (1979). Ramadan and diabetes (letter). British Medical Journal ii, 46-47.

Bargen-Lockner, C., Hahn, P. \& Wittmann, B. (1981). Plasma carnitine in pregnancy. American Journal of Obstetrics and Gynecology 140, 412-414.

Bissenden, J. G., Scott, P. H., King, J., Hallum, J., Mansfield, H. W. \& Wharton, B. A. (1981). Anthropometric and biochemical changes during pregnancy in Asian and European mothers having light for gestational age babies. British Journal of Obstetrics and Gynaecology 88, 998-1008.

Chapoy, P. R., Angelini, C., Brown, W. J., Stiff, J. E., Shug, A. L. \& Cederbaum, S. D. (1980). Systemic carnitine deficiency - a treatable inherited lipid-storage disease presenting as Reye's syndrome. New England Journal of Medicine 303, 1389-1394.

Eaton, P. M. \& Wharton, P. A. (1982). Sorrento study of nutrition in pregnancy. In Applied Nutrition, pp. 89-91 [E. C. Bateman, editor]. London: John Libbey.

Felig, P. \& Lynch, V. (1970). Starvation in human pregnancy; hypoglycemia, hypoinsulinemia, and hyperketonemia. Science 170, 990992.

Frost, G. \& Pirani, S. (1987). Meal frequency and nutritional intake during Ramadan: a pilot study. Human Nutrition: Applied Nutrition 41A, 47-50.

Maughan, R. A. (1982). A simple, rapid method for the determination of glucose, lactate, pyruvate, alanine, 3hydroxybutyrate and acetoacetate on a single $20 \mu \mathrm{l}$ blood sample. Clinica Chimica Acta 122, 231-240.

Prentice, A. M., Prentice, A., Lamb, W. H., Lunn, P. G. \& Austin, S. (1983). Metabolic consequences of fasting during Ramadan in pregnant and lactating women. Clinical Nutrition 37C, 283-294.

Secombe, D. W., Hahn, P. \& Novak M. (1978). The effect of diet and development of blood levels of free and esterified carnitine in the rat. Biochimica et Biophysica Acta 528, 483-487.

Thompson, A. M., Billewicz, W. Z. \& Hytten, F. E. (1968). The assessment of fetal growth. Journal of Obstetrics and Gynaecology of the British Commonwealth 75, 903916.

Tyson, J. E., Austin, K., Farinholt, J. \& Fiedler, J. (1976). Endocrine - metabolic response to acute starvation in human gestation. American Journal of Obstetrics and Gynecology 125, 1073-1084.

Viegas, O. A. C., Cole, T. J. \& Wharton, B. A. (1987). Impaired fat deposition in pregnancy: an indicator for nutritional intervention. American Journal of Clinical Nutrition 45, $23-28$. 